To illustrate the method, we consider a $\delta$ Scuti star for which several frequencies are observed, for instance $\nu_{0,1}, \nu_{0,2}, \nu_{0,3}$ (fundamental radial mode and first and second radial overtones) and the frequency of a "mixed mode" $\nu_{G 1}$ (see Unno et al., 1989). The accuracy on the frequency measurements is high $(0.1 \mu \mathrm{Hz})$, but the uncertainty on the position of the star in the H-R diagram (luminosity and effective temperature) can be important.

By means of theoretical models it is possible to estimate the unknown parameters of the star (mass, age, composition, physics) provided a sufficient number of observables (frequencies) is available. However as shown by Brown et al. (1994) and by Lebreton et al. (1994), some of the observables are not independent and the set of observables has to be carefully chosen. By choosing as independent observables $\nu_{0,1}, \nu_{0,3} / \nu_{0,1}$ and $\nu_{G 1}$ we try to solve for the most important physical unknown, the overshooting parameter $O_{v}$. We find that, with the actual precision on the measured frequencies, $O_{v}$ could be determined with a precision of $20 \%\left(0.03 H_{p}\right.$, where $H_{p}$ is the pressure scale-height) which is quite good since presently $O_{v}$ is estimated to be $0.15 \pm 0.15 \mathrm{H}_{p}$.

Unfortunately, the uncertainty on the helium abundance of those stars is high since it cannot be obtained by observations. So, in order to get estimates of both the overshooting distance and the helium abundance, it will be necessary either to observe more frequencies for a given star, or to observe several stars in the same cluster in order to increase the number of independent observables with respect to the number of unknown parameters. We have found that disregarding effects of fast rotation, for these stars, would lead to a misidentification of the modes.

Finally, we consider the effect of fast rotation. Fast rotation induces a frequency shift in the oscillation spectrum (Saio, 1981; Dziembowski \& Goode, 1993; Soufi et al., 1994). If this shift was neglected in the previous example, this would lead to erroneous values of the overshooting parameter. Rotation also modifies the position of a star in the H-R diagram with respect to a non-rotating star. The displacement in the $\mathrm{H}-\mathrm{R}$ diagram has been estimated by Maeder and Peytremann $(1970,1972)$.

\title{
16. Observations of deep-seated structure in the stellar winds of OB stars (R. K. Prinja)
}

High-resolution, time-resolved spectroscopy in both optical and UV wavebands has shown that the outer layers of luminous $O B$ stars vary on time scales of hours-days. Spectroscopic monitoring with the IUE satellite provides evidence that the stellar winds of luminous, hot stars are not smooth and steady, but are frequently disrupted by the presence of time-dependent structures. In addition, variability is often present in optical photospheric 
line profiles; these variations are likely due to the influence of photospheric velocity fields, especially those from one or more modes of nonradial pulsation (NRP). The process (or processes) responsible for the formation of time-dependent wind structure is (are) not known. Issues concerning potential connections between NRPs, variations at the base of the outflow, and the development of wind structure pose some of the greatest challenges to our understanding of mass-loss via radiatively driven stellar winds.

We have recently gathered UV data from IUE plus high-quality groundbased spectroscopy in order to constrain the formation mechanism of wind structure via the key issue of the location in the stellar wind at which structure - as diagnosed by variability -is first detectable. An important place to search for such deep-seated variability is in the optical spectrum, where all the wind features (e.g. $\mathrm{H} \alpha$, He I $\lambda 5876$ ) are due to recombination and hence preferentially formed in the high density regions of the wind; where extended time series of high $\mathrm{S} / \mathrm{N}$ spectra can be readily obtained. We have obtained extended, high $\mathrm{S} / \mathrm{N}(\sim 200)$ optical time series observations of He I $\lambda 5876$ and $\mathrm{H} \alpha$ for the extreme O supergiant, HD 152408 (Prinja \& Fullerton, 1994). Systematic variability is evident in the $\mathrm{P}$ Cygni absorption trough of $\mathrm{He}$ I $\lambda 5876$ at velocities as low as $-50 \mathrm{~km} / \mathrm{s}$.

In a separate study, Massa, Prinja \& Fullerton (1994), have carried out ultraviolet spectroscopy of the intermediate luminosity B-type supergiant, HD 64760. Continuous wind activity is documented over 6 consecutive days, with unambiguous evidence for the presence of structures deep in the supersonic wind. Substantial variability evident in the Si III $\lambda 1300 \AA$ triplet lines, which correlates with the strongest perturbations in the wind-formed resonance lines, provides firm evidence for a link between wind activity and the subsonic photospheric layers of the atmosphere. The observations also point to the long term, large scale spatial coherence of the wind structures.

Acknowleagment: I thank all participants of the meeting for their attendance and their contributions. My apologies go to all poster presenters, because their contributions could not be included in these proceedings due to limited space. I thank the members of the Scientific Organizing Committee, the Secretary General of the IAU Jaqueline Bergeron, and Monique and Julie in the IAU Paris Office, who all provided invaluable help and support during the last two years. And finally I am grateful to J.O. Stenflo who suggested that I should organize this Joint-Discussion Meeting. My work was supported in part by the NSF grant AST-9315112 to USC, that of P. Demarque and his collaborators in part by the NASA grants NAGW-2531 and NAG5-1486 to Yale University. 


\section{References}

Accetta, F.S., Krauss, L.M. \& Romanelli, P. 1990, Phys. Let., B248, 146.

Andersen, B.N. \& Domingo, V., IAU Symposium 123, (J.Christensen-Dalsgaard \& S.Frandsen eds.), Reidel, p. 67.

Antia, H.M., Basu, S., 1994, Astron. Astrophys. Suppl. Ser, 107, 421.

Antia, H.M., Chitre, S.M., 1995, Astrophys. J., in press.

Appourchaux, T. \& Andersen, B.N., 1990, Sol.Phys. 128, 91.

Appourchaux, T., Martin, D. \& Telljohann, U., 1992, SPIE 1679, 200.

Bachmann, K.T. \& Brown, T.J., 1993, Astrophys. J., 411, L45.

Bachmann, K.T., Duvall, T.L. Jr., Harvey, J.W. \& Hill, F., 1995, in Helio- and Asteroseismology from the Earth and Space: A.S.P. Conf. Series 76 (R.K. Ulrich, E.J. Rhodes Jr., \& W. Däppen, eds.), 156.

Bahcall, J. N. \& Loeb, A. 1990, Astrophys. J., 360, 267.

Bahcall, J. N. \& Pinsonneault, M. H. 1992a, Rev. Mod. Phys., 64, 885.

Bahcall, J. N. \& Pinsonneault, M. H. 1992b, Astrophys. J., 395, L119.

Basu, S., Antia, H. M., 1994a, J. Astrophys. Astron., 15, 143.

Basu, S., Antia, H. M., 1994b, Mon. Not. Roy. Astron. Soc., 269. 1137

Basu, S., Thompson, M. J., 1994, submitted.

Bradley, P. A. 1993, Ph.D. thesis, University of Texas.

Brodsky, M. A. \& Vorontsov, S. V., 1993, Astrophys. J., 409, $455-464$.

Brown T.M., Christensen-Dalsgaard J., Weibel-Mihalas B., Gilliland R., 1994, Astrophys. J. 427, 1013.

Brown, T.M. \& Morrow, C.A., 1987, in The internal Solar Angular Velocity, (B.R.Durney \& S.Sofia eds.), Reidel, p. 7.

Canuto, V. M. \& Mazzitelli, I. 1991, Astrophys. J., 370, 295.

Chaboyer, B., 1993, Ph.D. thesis, Yale University.

Chaboyer, B., Demarque, P. \& Pinsonneault, M. H. 1995a, Astrophys. J., March 10 issue.

Chaboyer, B. ,Demarque, Guenther,D.B. \& Pinsonneault,M.H. 1995b, Astrophys. J., June 10 issue.

Christensen-Dalsgaard, J. \& Däppen, W., 1992. Astron. Astrophys. Rev., 4, $267-361$.

Christensen-Dalsgaard, J., Duvall, T. L., Gough, D. O., Harvey, J. W. \& Rhodes, E. J., 1985, Nature, 315, $378-382$.

Christensen-Dalsgaard, J., Gough, D. O. \& Thompson, M. J., 1989, Mon. Not. Roy. Astron. Soc., 238, $481-502$.

Christensen-Dalsgaard, J., Gough, D. O. \& Pérez Hernández, F., 1988, Mon. Not. Roy. Astron. Soc., 235, $875-880$.

Christensen-Dalsgaard, J., Gough, D.O. \& Thompson, M.J. 1991, Astrophys. J., 378, 413

Christensen-Dalsgaard, J., Proffitt, C.R. \& Thompson, M.J. 1993, Astrophys. J., 403, L75.

Christensen-Dalsgaard, J., Proffitt, C.R., Thompson, M.J., 1993, Astrophys. J., 408, L75.

Clemens, J. C. 1994, Ph.D. thesis, University of Texas.

Däppen, W., Anderson, L. S. \& Mihalas, D., 1987, Astrophys. J. 319, 195.

Däppen, W., 1992, Rev. Mexicana Astron. Astrof. 23, 1144.

Demarque, P., Krauss, L.M., Guenther, D.B. \& Nydam, D. 1994, Astrophys. J., 437, 870.

Deubner, F.L., 1975, Astron. Astrophys., 44, 371.

Dziembowski W.A. \& Goode P.R., 1992, Astrophys. J. 394, 670.

Dziembowski, W. A., Pamyatnykh, A. A. \& Sienkiewicz, R., 1992, Acta Astron., 42, 5 15.

Eff-Darwich, A., Roca Cortes \& van der Raay, H.B., 1995, in Helio- and Asteroseismology from the Earth and Space: A.S.P. Conf. Series 76 (R.K. Ulrich, E.J. Rhodes Jr., \& W. Däppen, eds.), 47.

Eggleton, P. P., Faulkner, J. \& Flannery, B. P., 1973, Astron. Astrophys., 23, 325 - 330.

Elsworth, Y., Howe, R., Isaak, G.R., McLeod, C.P., Miller, B.A., New, R, Speake, C.C. \& Wheeler, S.J., 1994, Astrophys. J., 434, 801.

Elsworth, Y., Howe, R., Isaak, G.R., McLeod, C.P., Miller, B.A., Wheeler, S.J., New, R., 
\& Gough, D.O., 1995a, in Helio- and Asteroseismology from the Earth and Space: A.S.P. Conf. Series 76 (R.K. Ulrich, E.J. Rhodes Jr., \& W. Däppen, eds.), 43.

Elsworth, Y., Howe, R., Isaak, G.R., McLeod, C.P., Miller, B.A., Wheeler, S.J. \& New, R., 1995b, in Helio- and Asteroseismology from the Earth and Space: A.S.P. Conf. Series 76 (R.K. Ulrich, E.J. Rhodes Jr., \& W. Däppen, eds.), 51.

Endal, A.S. \& Sofia, S. 1978, Astrophys. J., 220, 279.

Endal, A.S. \& Sofia, S. 1981, Astrophys. J., 243, 625.

Fossat, E., Loudagh, S., Gelly, B., Grec, G., Pantel, A., Provost, J., Berthomieu, G., Ehgamberdiev, S. Khalikov, S. Lazrek, M., Palle, P.L. \& Regulo, C., 1995, in Helioand Asteroseismology from the Earth and Space: A.S.P. Conf. Series 76 (R.K. Ulrich, E.J. Rhodes Jr., \& W. Däppen, eds.), 24.

Genovese, C.R., Stark, P.B. \& Thompson, M.J., 1995, Astrophys. J., in press.

Gough, D. O., 1984. Phil. Trans. R. Soc. London, Ser. A, 313, $27-38$.

Gough, D. O. \& Thompson, M. J., 1991. in Solar interior and atmosphere, eds Cox, A. N., Livingston, W. C. \& Matthews, M., p. 519 - 561, Space Science Series, University of Arizona Press.

Gough, D. O. \& Vorontsov, S.V., 1995. Mon. Not. Roy. Astron. Soc., in press.

Gough, D.O, Kosovichev, A.G. \& Toutain, T., 1995, in Helio- and Asteroseismology from the Earth and Space: A.S.P. Conf. Series 76 (R.K. Ulrich, E.J. Rhodes Jr., \& W. Däppen, eds.), 176.

Guenther, D.B. 1994, Astrophys. J., 422, 400.

Guenther, D. B., Demarque, P., Kim, Y.-C. \& Pinsonneault, M. H. 1992a, Astrophys. J., 387,372 .

Guenther, D. B., Demarque, P., Pinsonneault, M. H. \& Kim, Y.- C. 1992b, Astrophys. J., 392, 328.

Guenther, D. B., Pinsonneault, M. H. \& Bahcall, J. N. 1993, Astrophys. J., 418, 469.

Guenther, D.B., Sills, K., Demarque, P. \& Krauss, L. 1995, Astrophys. J., May 20 issue.

Guzik, J.A., Cox, A.N., 1993, Astrophys. J., 411, 394.

Hill, F., 1995, in Helio- and Asteroseismology from the Earth and Space: A.S.P. Conf. Series 76 (R.K. Ulrich, E.J. Rhodes Jr., \& W. Däppen, eds.), 484.

Hummer, D. G. \& Mihalas, D., 1988, Astrophys. J. 331, 794.

Jimenez, A., Perez Hernandez, F., Claret, A., Palle, P.L., Regulo, C. \& Roca Cortes, T., 1994, Astrophys, J., 435, 874.

Jimenez, A., Perez Hernandez, F., Claret, A., Palle, P.L., Regulo, C. \& Roca Cortes, T., 1995, in Helio- and Asteroseismology from the Earth and Space: A.S.P. Conf. Series 76 (R.K. Ulrich, E.J. Rhodes Jr., \& W. Däppen, eds.), 284.

Kahn, F. D., 1961: Astrophys. J. 134, 343.

Korzennik, S.G., Cacciani, A., Rhodes, E.J., Jr. \& Ulrich, R.K., 1990, in Progress of Seismology of the Sun and Stars, Lecture Notes in Physics, 367, (Y. Osaki \& H. Shibahashi, eds.), 341.

Korzennik, S.G., Rhodes, E.J., Jr., Johnson, N.M. \& Rose, P., 1995, in Helio- and Asteroseismology from the Earth and Space: A.S.P. Conf. Series 76 (R.K. Ulrich, E.J. Rhodes Jr., \& W. Däppen, eds.), 12.

Kosovichev, A. G., Christensen-Dalsgaard, J., Däppen, W., Dziembowski, W. A., Gough, D. O. \& Thompson, M. J., 1992, Mon. Not. Roy. Astron. Soc., 259, 536 - 558.

Krauss, L. M. \& White, M. 1992, Astrophys. J., 394, 385.

Kurucz,R.L.1991, in Stellar Atmospheres: Beyond Classical Models, (eds. L.Crivellari, I.Hubeny, D.G.Hummer), Kluwer, 440.

Lebreton Y., Michel E., Goupil M.J., Baglin A., Fernandes J., 1994, IAU Symp. 166.

Leighton, R.B., Noyes, R.W. \& Simon, G.W., 1962, Astrophys. J., 135, 474.

Libbrecht, K. G., Woodard, M. F. \& Kaufman, J. M. 1990, Astrophys. J. Suppl., 74, 1129.

Loudagh, S. et al.1993, Astron. Astrophys., 275, L25.

Maeder A. \& Peytreman E., 1970, Astron. Astrophys. 7, 120.

Maeder A. \& Peytreman E., 1972, Astron. Astrophys. 21, 279. 
Massa, D., Prinja R. K. \& Fullerton, A. W. 1994, Astrophys. J., submitted.

Palle, P.L., 1995, in Helio- and Asteroseismology from the Earth and Space: A.S.P. Conf. Series 76 (R.K. Ulrich, E.J. Rhodes Jr., \& W. Däppen, eds.), 239.

Patron, J., Hill, F., Rhodes, E.J., Jr., Korzennik, S.G. \& Cacciani, 1995, in Helio- and Asteroseismology from the Earth and Space: A.S.P. Conf. Series 76 (R.K. Ulrich, E.J. Rhodes Jr., \& W. Däppen, eds.), 208.

Pérez Hernández, F. \& Christensen-Dalsgaard, J., 1994. Mon. Not. Roy. Astron. Soc., $475-492$.

Pinsonneault, M. H., Kawaler, S. D., Sofia, S. \& Demarque, P. 1989, Astrophys. J., 338, 424

Pinsonneault, M. H., Kawaler, S. D. \& Demarque, P. 1990, Astrophys. J. Suppl., 74, 501.

Prinja, R. K. \& Fullerton, A. W. 1994, Astrophys. J. 426, 345.

Proffitt, C. R. \& Michaud, G. 1991, Astrophys. J., 380, 238.

Regulo, C., Jimenez, A., Palle, P.L., Perez Hernandez, F. \& Roca Cortes, T., 1994, Astrophys. J., 434, 384.

Rhodes, E.J., Jr, Cacciani, A., Korzennik, S. et al., 1990, Astrophys. J. 351, 687.

Rhodes, E.J., Jr., Cacciani, A., Korzennik, S.G. \& Ulrich, R.K., 1993, Astrophys. J., 406, 714.

Rhodes, E.J., Jr., Johnson, N.M., Rose, P.J., Korzennik, S.G. \& Cacciani, A., 1995, in Helio- and Asteroseismology from the Earth and Space: A.S.P. Conf. Series 76 (R.K. Ulrich, E.J. Rhodes Jr., \& W. Däppen, eds.), 227.

Rogers, F. J., 1981, Phys. Rev. A24, 1531.

Rogers, F. J., 1986, Astrophys. J. 310, 723.

Rogers, F. J., 1994, in The Equation of Sate in Astrophysics IAU Colloquium 147 (eds. G. Chabrier \& E. Schatzman), Cambridge University Press, 16.

Rogers, F.J \& Iglesias, C. A. 1994, Science, 263, 50.

Saio H., 1981, Astrophys. J. 244, 299.

Schou, J. \& Tomczyk, S., 1995, in Helio- and Asteroseismology from the Earth and Space: A.S.P. Conf. Series 76 (R.K. Ulrich, E.J. Rhodes Jr., \& W. Däppen, eds.), 448.

Sofia, S., Heaps, W. \& Twigg, L. 1994, Astrophys. J., 427, 1048.

Soufi F., Goupil M.J., Dziembowski W.A., Sienkiewicz R., 1995, Astron. Astrophys., submitted.

Tassoul, M., 1980, Astrophys. J. Suppl., 43, $469-490$.

Tomczyk, S., Streander, K., Card, G., Elmore, D., Hull, H. \& Cacciani, A., 1995a, Solar Physics, in press.

Tomczyk, S., Schou, J. \& Thompson, M.J., 1995b, Astrophys. J. Lett, in press.

Toutain, T., 1995, in Helio- and Asteroseismology from the Earth and Space: A.S.P. Conf. Series 76 (R.K. Ulrich, E.J. Rhodes Jr., \& W. Däppen, eds.), 34.

Toutain, Th. \& Fröhlich, C.1992, Astron. Astrophys., 257, 287.

Toutain, T. \& Kosovichev, A., 1994, Astron. Astrophys. 284, 265.

Unno W., Osaki Y., Ando H., Saio H., Shibahashi H., 1989, Nonradial oscillations of stars (2nd edition), Univ. of Tokyo Press.

Vandakurov, Yu. V., 1967. Astron. Zh., 44, 786 - 797. (English translation: Soviet Astronomy A.J., 11, $630-638$ ).

Vorontsov, S. V., Baturin, V. A. \& Pamyatnykh, A. A., 1991, Nature, 349, 49 - 51.

Weidemann, V. 1990, Astron. Astrophys. 28, 103.

Winget, D. E. 1988, in IAU Symp. 123, Advances in Helio- and Asteroseismology, ed. J. Christensen-Dalsgard and S. Frandsen, (Dordrecht: Reidel), 305.

Winget, D. E. et al. 1991, Astrophys. J. 378, 326.

Winget, D. E. et al. 1994, Astrophys. J. 430, 839.

Woodard, M.F., Kuhn, J.R., Murray, N. \& Libbrecht, K.G., 1991, Astrophys. J., 373, L81.

Woodard, M.F. \& Libbrecht, K.G., 1993a, Astrophys. J., 402, L77.

Woodard, M.F. \& Libbrecht, K.G., 1993b, Science, 260, 1778.

Zhugzhda, Y. D., Stix, M., 1994: Astron. Astrophys., in press. 